\title{
Effects of axial vibration on vertical zone-melting processing
}

\author{
C.W. Lan \\ Chemical Engineering Department, National Taiwan University, 10617, Taipei, Taiwan
}

Received 20 March 1999; received in revised form 9 August 1999

\begin{abstract}
Vibration can be an effective way for controlling heat flow and the growth interface during crystal growth. To better understand the role of axial vibration in vertical zone melting, an important process for crystal growth and purification, computer simulation is performed. For the zone melting of a 2-cm GaAs crystal in a quartz ampoule, simulation results show that an axial vibration, especially at high frequency, can significantly affect the flows and further the growth interface. The concave growth front due to buoyancy convection can be also inverted easily to a convex one. The effects of vibration frequency are further illustrated, and the validity of the high-frequency model is examined. For GaAs, the resonance frequency is found at about $0.5 \mathrm{~Hz}$, where significant flow oscillation and severe periodic growth and remelting can be induced. (C) 2000 Elsevier Science Ltd. All rights reserved.
\end{abstract}

Keywords: Vibration; Zone-melting; Interface control; Buoyancy convection; Growth rate; Thermovibration

\section{Introduction}

The control of melt flow, the interface shape, and the growth rate is important in crystal growth processes. Especially, for crystal growth in an ampoule, a flat or convex growth front is usually required to minimize the parasitic nucleation of grains from the ampoule wall [1]. The convection and its associated heat and mass transfer are key factors for the interface control. For purification, mixing is also important on its efficiency. In general, tuning the furnace configurations to control the melt flow and thus the interface shape and mixing is typical, but it is usually difficult and less flexible [2,3]. Therefore, using external forces to control crystal growth has been widely adopted [415]. Magnetic fields (e.g., [4-6]) and rotations [7-9],

E-mail address: lan@ruby.che.ntu.edu.tw (C.W. Lan). steady rotation or accelerated crucible rotation technique (ACRT) [8], are two typical approaches. Recently, vibration has also been found useful in floating-zone (FZ) [10,11] and vertical Bridgman crystal growth [13-15]. Some theoretical study has also been performed (e.g., [11,15]). Contributions due to thermovibration and surface waves have also been discussed from the fluid mechanics point of view. However, some important issues for crystal growth, such as the interface control, effects of vibration frequency (especially near resonance), and the induced growth rate oscillation, etc., have been ignored.

For crystal growth in an ampoule, the melt is often confined by the rigid ampoule wall and the deformable growth interface. As the ampoule vibrates, the heat flow as well as the interface also vibrates. Although the melt can be treated as an incompressible fluid, its thermal expansion coefficient is not zero. Accordingly, the vibration, which works 


\begin{tabular}{|c|c|c|c|}
\hline \multicolumn{4}{|c|}{ Nomenclature } \\
\hline$a$ & width parameter in $T_{\text {eff }}$ & $U_{\mathrm{amp}}$ & ampoule pulling speed \\
\hline$b_{\mathrm{v}}$ & vibration amplitude & $v$ & $z$-component of velocity \\
\hline$B i$ & Biot number, $h R_{\mathrm{c}} / k_{\mathrm{m}}$ & $w$ & width of heating profile \\
\hline$C_{\mathrm{p}}$ & specific heat & $w_{r}$ & $r$-component of pulsation velocity \\
\hline$e_{z}$ & unit vector in $z$-direction & $w_{z}$ & $z$-component of pulsation velocity \\
\hline$f_{\mathrm{v}}$ & vibration frequency & $z$ & cylindrical coordinate \\
\hline$g_{0}$ & gravitational acceleration & $z_{\mathrm{p}}$ & position of peak heater temperature \\
\hline$\Delta H$ & heat of fusion & & \\
\hline$h$ & heat transfer coefficient & \multicolumn{2}{|c|}{ Greek symbols } \\
\hline$\Delta h_{\mathrm{c}}$ & $\begin{array}{l}\text { deflection of growth front, }\left.h_{\mathrm{c}}\right|_{r=R_{\mathrm{c}}}- \\
\left.h_{\mathrm{c}}\right|_{\gamma=0}\end{array}$ & $\begin{array}{l}\alpha \\
\beta_{\mathrm{T}}\end{array}$ & $\begin{array}{l}\text { thermal diffusivity } \\
\text { thermal expansion coefficient }\end{array}$ \\
\hline$h_{\mathrm{c}}$ & height of growth front & $\varepsilon$ & emissivity \\
\hline$h_{\mathrm{f}}$ & height of feed front & $\eta$ & relative amplitude of vibration, $b_{\mathrm{v}} \Omega_{\mathrm{v}}^{2} / g_{0}$ \\
\hline$k$ & thermal conductivity & $v_{\mathrm{m}}$ & kinematic viscosity, $\mu_{\mathrm{m}} / \rho_{\mathrm{m}}$ \\
\hline$L$ & length of ampoule & $\mu_{\mathrm{m}}$ & viscosity \\
\hline$n$ & unit normal vector & $\rho$ & density \\
\hline $\operatorname{Pr}$ & Prandtl number, $v_{\mathrm{m}} / \alpha_{\mathrm{m}}$ & $\sigma$ & Stefan-Boltzmann constant \\
\hline$r$ & cylindrical coordinate & $\psi$ & stream function \\
\hline$R a_{\mathrm{T}}$ & $\begin{array}{l}\text { thermal Rayleigh number, } \\
\beta_{\mathrm{T}} R_{\mathrm{c}}^{3} g_{0} T_{\mathrm{m}} / \nu_{\mathrm{m}} \alpha_{\mathrm{m}}\end{array}$ & $\begin{array}{l}\psi_{\mathrm{w}} \\
\omega\end{array}$ & $\begin{array}{l}\text { pulsation stream function } \\
\text { vorticity }\end{array}$ \\
\hline$R a_{\mathrm{v}}$ & $\begin{array}{lll}\text { vibrational } & \text { Rayleigh } & \text { number, } \\
\left(\beta_{\mathrm{T}} b_{\mathrm{v}} \Omega_{\mathrm{v}} T_{\mathrm{m}} R_{\mathrm{c}}\right)^{2} /\left(2 v_{\mathrm{m}} \alpha_{\mathrm{m}}\right) & \end{array}$ & $\Omega_{\mathrm{v}}$ & angular vibration frequency \\
\hline Rad & radiation number, $\sigma \varepsilon_{\mathrm{amp}} T_{\mathrm{m}}^{3} R_{\mathrm{c}} / k_{\mathrm{m}}$ & Superscript & \\
\hline$R_{\mathrm{c}}$ & radius of crystal & $*$ & dimensionless variables \\
\hline$R_{\mathrm{amp}}$ & radius of ampoule & & \\
\hline St & Stefan number, $\rho_{\mathrm{c}} \Delta H / C_{\mathrm{p}_{\mathrm{m}}} T_{\mathrm{m}}$ & Subscripts & \\
\hline$t$ & time & a & averaged value \\
\hline$T$ & temperature & amp & ampoule \\
\hline$T_{\text {eff }}$ & effective heater temperature & f & feed \\
\hline$T_{\mathrm{m}}$ & melting point & $\mathrm{c}$ & crystal \\
\hline$T_{\mathrm{p}}$ & peak heater temperature & $\mathrm{m}$ & melt \\
\hline$T_{\infty}$ & background ambient temperature & $\max$ & maximum \\
\hline$u$ & $r$-component of velocity & $\min$ & minimum \\
\hline$U_{\mathrm{h}}$ & heater moving speed & & \\
\hline
\end{tabular}

like a gravitational field, can induce periodic buoyancy flow. This is the so-called the thermovibration effect. Furthermore, since the growth interface vibrates as well, it may also affect the bulk flow, especially when the melt and solid densities are different. However, this is believed to be a secondary effect because the amplitude of interface vibration is usually much smaller than the applied one. Interestingly, when the vibration frequency is high enough, the heat flow becomes harmonic. Hence, a Boussinesq-type approximation (time-averaged heat flow) can be obtained by taking a time average over one vibration cycle. The vibrating interface can be treated by including the Schlichting boundary condition [16] instead of the no-slip one. Such an approximation greatly simplifies the numerical solution of the original time-dependent problem that contains two very different time scales. However, if the frequency is away from the high-frequency limit, the approximation breaks down due to the resonance effect, and the full numerical solution of the stiff problem is necessary. Indeed, for crystal growth applications, the regime of operation is particularly important. Operation near the resonance can induce severe flow unsteadiness leading to growth striations

So far, the numerical study of vibration in crystal growth is still very few. Uspenskii and Favier [15] used the high-frequency approximation to investigate the possible damping effects due to vibration for both vertical and horizontal Bridgman configurations. Lyubimov et al. [11,17] also used a similar approach to investigate the effects of different vibration mechanisms for the FZ system. They observed that the surface 
wave and thermovibration mechanisms were dominant in the melt, while the Schlichting effect was much smaller. Furthermore, the suppression of thermocapillary flow by axial vibration was found possible. The general applications of vibration for FZ and Czochralski methods were further illustrated by Lyubimov et al. [11], again, for the high-frequency limit. Nevertheless, in the previous study, the effects of vibration on the interface shape and the growth rate were not incorporated. The induced growth oscillation has not been discussed as well. For crystal growth simulation in a self-consistence manner, the moving interfaces need to be considered simultaneously with the field variables. Of course, the moving boundary also imposes an additional challenge task to numerical simulation. Therefore, in this study, we use vertical zone melting (VZM), an important process for crystal growth and purification [18], to illustrate the significance of axial vibration with the frequency ranging from 0.1 to $100 \mathrm{~Hz}$. The high-frequency limit and its breakdown due to the resonance are further illustrated.

In this paper, using the zone-melting of 2-cm GaAs in a quartz ampoule as a model system, we will first illustrate how the natural convection can be suppressed by thermovibration leading to an interface inversion, which is favorable for crystal growth. The critical vi-

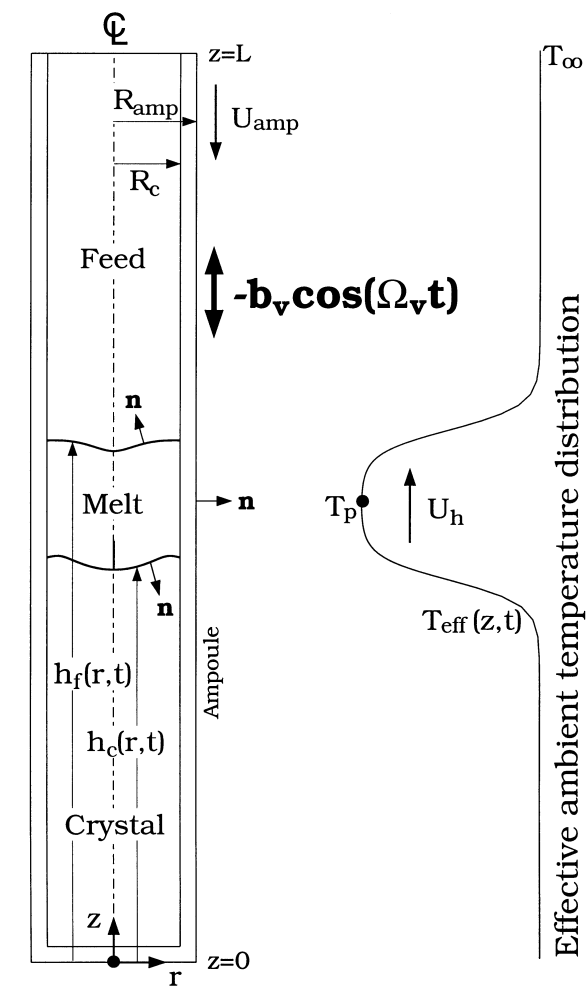

Fig. 1. Schematic sketch of vertical zone-melting (VZM). bration intensity for the interface inversion is obtained. The validity of the high-frequency limit is then examined by fully time-dependent calculations. From there, the frequency effects and the onset of resonance, as well as the induced growth oscillation, are then discussed. In the next section, the governing equations for both general and high-frequency models are presented first. The numerical solution of these equations is brief described. The results and discussion is devoted in Section 3, followed by brief conclusions and comments in Section 4.

\section{Models and numerical solution}

The schematic of the VZM crystal growth used in this study is depicted in Fig. 1. The furnace is described by an effective heating profile $T_{\text {eff }}(z, t)$, which is specified in modeling. To start crystal growth from a stationary state, this profile can be moved upward at speed $U_{\mathrm{h}}$. The vibration is applied in the axial direction with an amplitude $b_{\mathrm{v}}$ and angular frequency $\Omega_{\mathrm{v}}$ (or frequency $f_{\mathrm{v}}$ in $\mathrm{Hz}$ ); $\Omega_{\mathrm{v}}=2 \pi f_{\mathrm{v}}$. To simplify the calculation, this effect is incorporated into the gravitational acceleration, i.e., $g=g_{0}+b_{\mathrm{v}} \Omega_{\mathrm{v}}^{2} \cos \left(\Omega_{\mathrm{v}} t\right)$; the acceleration is a second derivative of the distance. Usually, near the middle of the ampoule, a steadystate growth can be reached in $20 \mathrm{~min}$ [9]. Therefore, if a stable growth is possible, the heating profile is kept stationary and the ampoule is moving downward at

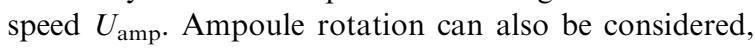
but it is beyond the scope of present study. The system is assumed axisymmetric, so that the flow and temperature fields, as well as the growth front (the melt/crystal interface, $\left.h_{\mathrm{c}}(r, t)\right)$, and the feed $/$ melt interface, $h_{\mathrm{f}}(r, t)$, are represented in a cylindrical coordinate system $(r, z)$.

The melt is further assumed incompressible and Newtonian, while the flow is laminar. The Boussinesq approximation is also adopted. Dimensionless variables are defined by scaling length by $R_{\mathrm{c}}$, time $t$ by $R_{\mathrm{c}}^{2} / \alpha_{\mathrm{m}}$, velocity by $\alpha_{\mathrm{m}} / R_{\mathrm{c}}$, and temperature by the melting point $T_{\mathrm{m}}$, where $\alpha_{\mathrm{m}}$ is the thermal diffusivity of the melt. For the convenience of representation, all the variables defined afterwards with a superscript * are dimensionless unless otherwise stated. The governing equations for the time-dependent heat transfer and fluid flow in terms of dimensionless stream function $\psi^{*}$, vortivity $\omega^{*}$, and temperature $T^{*}$ can be written as the following:

Equation of motion

$-\frac{\partial \omega^{*}}{\partial t^{*}}+\frac{\partial}{\partial r^{*}}\left(\frac{\omega^{*}}{r^{*}} \frac{\partial \psi^{*}}{\partial z^{*}}\right)-\frac{\partial}{\partial z^{*}}\left(\frac{\omega^{*}}{r^{*}} \frac{\partial \psi^{*}}{\partial r^{*}}\right)$ 


$$
\begin{aligned}
& +\operatorname{Pr}\left[\frac{\partial}{\partial r^{*}}\left(\frac{1}{r^{*}} \frac{\partial}{\partial r^{*}}\left(r^{*} \omega^{*}\right)\right)+\frac{\partial}{\partial z^{*}}\left(\frac{1}{r^{*}} \frac{\partial}{\partial z^{*}}\left(r^{*} \omega^{*}\right)\right)\right] \\
& \quad-\operatorname{Pr} \operatorname{Ra}\left[1+\eta \cos \left(\Omega_{\mathrm{v}}^{*} t^{*}\right)\right] \frac{\partial T^{*}}{\partial r^{*}} \\
& \quad=0 .
\end{aligned}
$$

Stream equation

$$
\frac{\partial}{\partial z^{*}}\left(\frac{1}{r^{*}} \frac{\partial \psi^{*}}{\partial z^{*}}\right)+\frac{\partial}{\partial r^{*}}\left(\frac{1}{r^{*}} \frac{\partial \psi^{*}}{\partial r^{*}}\right)+\omega^{*}=0 .
$$

Energy equation

$$
\begin{aligned}
& -r^{*} \frac{\partial T^{*}}{\partial t^{*}}-\frac{\partial}{\partial r^{*}}\left(r^{*} u^{*} T^{*}\right)-\frac{\partial}{\partial z^{*}}\left(r^{*} v^{*} T^{*}\right) \\
& +\frac{\partial}{\partial z^{*}}\left(r^{*} \alpha_{i}^{*} \frac{\partial T^{*}}{\partial z^{*}}\right)+\frac{\partial}{\partial r^{*}}\left(r^{*} \alpha_{i}^{*} \frac{\partial T^{*}}{\partial r^{*}}\right)=0, \\
& \quad i=\mathrm{m}, \mathrm{f}, \mathrm{c}, \mathrm{amp} .
\end{aligned}
$$

In the above equations, $R a_{\mathrm{T}}$ and $\eta$ are the thermal Rayleigh number and the relative amplitude of gravity modulation, respectively, defined as follows:

$R a_{\mathrm{T}} \equiv \frac{\beta_{\mathrm{T}} R_{\mathrm{c}}^{3} g_{0} T_{\mathrm{m}}}{\nu_{\mathrm{m}} \alpha_{\mathrm{m}}} ; \quad \eta \equiv \frac{b_{\mathrm{v}} \Omega_{\mathrm{v}}^{2}}{g_{0}}$,

where $\beta_{\mathrm{T}}$ is the thermal expansion coefficient, $g_{0}$ the normal gravitational acceleration, $v_{\mathrm{m}}$ the melt kinematic viscosity, and $\alpha_{i}$ the thermal diffusivity of phase $i$; $i$ represents the melt (m), the feed (f), the crystal (c), or the ampoule (amp). Also, the dimensionless thermal diffusivity is defined by $\alpha_{i}^{*}=\alpha_{i} / \alpha_{\mathrm{m}}$. The Prandtl number $\operatorname{Pr}$ is defined as usual; $\operatorname{Pr}=v_{\mathrm{m}} / \alpha_{\mathrm{m}}$. The stream function $\psi$ and vortivity $\omega$ (dimensional) are defined in terms of radial $(u)$ and axial $(v)$ velocities, respectively, as

$u=-\frac{1}{\rho_{\mathrm{m}} r} \frac{\partial \psi}{\partial z}, \quad v=\frac{1}{\rho_{\mathrm{m}} r} \frac{\partial \psi}{\partial r}$

and

$\omega=\frac{\partial u}{\partial z}-\frac{\partial v}{\partial r}$

Again, the dimensionless stream function $\psi^{*}$ is defined by scaling $\psi$ by $\rho_{\mathrm{m}} \alpha_{\mathrm{m}} R_{\mathrm{c}}$, while $\omega^{*}$ is defined by scaling $\omega$ by $\alpha_{\mathrm{m}} / R_{\mathrm{c}}^{2}$.

To solve the previous equations, boundary conditions are required. Most of the boundary conditions can be found elsewhere [19]. In brief, the no-slip boundary conditions are adopted for solid boundaries, where the density difference between solid and the melt is neglected. Since the melt velocity is usually much larger than the interface speed, such an approximation is believed to be reasonable. Heat transfer from the system to the ambient is governed by convection and radiation:

$$
\begin{aligned}
& -\left(\boldsymbol{n} \kappa_{\mathrm{amp}} \cdot \nabla^{*} T^{*}\right)=B i\left[T^{*}-T_{\mathrm{eff}}^{*}\left(z^{*}, t^{*}\right)\right] \\
& +\operatorname{Rad}\left[T^{* 4}-T_{\mathrm{eff}}^{*}\left(z^{*}, t^{*}\right)^{4}\right],
\end{aligned}
$$

where $\kappa_{\text {amp }}$ is the ratio of ampoule thermal conductivity to the melt, $B i \equiv h R_{\mathrm{c}} / k_{\mathrm{m}}$ the Biot number, and $R a d \equiv \sigma \varepsilon_{\mathrm{amp}} T_{\mathrm{m}}^{3} R_{\mathrm{c}} / k_{\mathrm{m}}$ the radiation number; $k_{\mathrm{m}}$ is the thermal conductivity of the melt, $\sigma$ the Stefan-Boltzmann constant, $\varepsilon_{\text {amp }}$ the ampoule emissivity. Furthermore, $T_{\text {eff }}(z, t)$ is the effective ambient temperature, which is described by a Fermi-like distribution:

$T_{\text {eff }}(z)=T_{\infty}+\frac{T_{\mathrm{p}}-T_{\infty}}{\exp \left(\left|z-z_{\mathrm{p}}(t)\right|-w\right) / a+1}$,

and

$z_{\mathrm{p}}(t)=z_{\mathrm{p} 0}+\int_{0}^{t} U_{\mathrm{h}} \mathrm{d} t$

where $T_{\mathrm{p}}$ and $T_{\infty}$ are the peak and background temperatures, respectively, the parameters $w$ and $a$ are related to the width of the distribution, $z_{\mathrm{p}}(t)$ is the position of $T_{\mathrm{p}}$, and $z_{\mathrm{p} 0}$ the initial position of $T_{\mathrm{p}}$. Again, the dimensionless $T^{*}$ is defined by scaling $T$ by $T_{\mathrm{m}}$. An example of this distribution is shown on the RHS of Fig. 1, which is similar to that measured from a typical zone heater furnace [20]. The top surface of the system is assumed adiabatic. A more realistic boundary condition can be used if necessary.

At the feed/melt and the melt/crystal interfaces, the Stefan boundary condition is used:

$$
\begin{aligned}
& \left.\kappa_{i}\left(\boldsymbol{n} \cdot \nabla^{*} T^{*}\right)\right|_{i}-\left.\left(\boldsymbol{n} \cdot \nabla^{*} T^{*}\right)\right|_{\mathrm{m}} \\
& \quad+\gamma S t\left(U_{\mathrm{amp}}^{*}+\frac{\partial h_{i}^{*}}{\partial t^{*}}\right) \boldsymbol{n} \cdot e_{z}=0, \quad i=\mathrm{f}, \mathrm{c},
\end{aligned}
$$

where $S t \equiv \rho_{\mathrm{c}} \Delta H / C_{\mathrm{p}_{\mathrm{m}}} T_{\mathrm{m}}$ is the Stefan number and $\gamma \equiv$ $\rho_{\mathrm{c}} / \rho_{\mathrm{m}}$ the density ratio of solid and the melt. As shown in Eq. (9), the higher the Stefan number (or heat of fusion), the smaller the interface velocity is affected by the heat flow.

The above governing equations and their associated boundary conditions can only be solved numerically. We have developed efficient finite-volume schemes using Newton's method with a solution tracking capability and the DASPK solver for time integration $[19,21]$ for solving these equations. Detailed description of the numerical method can be found elsewhere [19].

In most applications, the vibration frequency is usually in the order of 0.1 to $10^{3} \mathrm{~Hz}$. However, the time constant for fluid flow (for GaAs) is about $R_{\mathrm{c}}^{2} / v_{\mathrm{m}}$ 
being about $300 \mathrm{~s}$, while for heat transfer it is about $20 \mathrm{~s}\left(R_{\mathrm{c}}^{2} / \alpha_{\mathrm{m}}\right)$. For a steady growth to be achieved, 10 to $20 \mathrm{~min}$ is necessary in general. Therefore, for high frequency vibration, the solution of the full equations is extremely time consuming because the stepsize for integration needs to be smaller than the period of vibration, while the integration time is very long (longer than $10 \mathrm{~min}$ ) before a steady state could be achieved. In order to overcome the difficulty, we have adopted the high-frequency approximation [22] to previous equations by taking the time average over one period of vibration $\left(2 \pi / \Omega_{\mathrm{V}}\right)$. We assume that the frequency is high enough for the period to be small with respect to the hydrodynamic and thermal times. Also, the induced vibration amplitude of the field variables is assumed small. After the time average over the fast terms, as well as neglecting the higher order terms, the governing equation for the equation of motion is slightly changed:

$$
\begin{aligned}
& -\frac{\partial \omega_{a}^{*}}{\partial t^{*}}+\frac{\partial}{\partial r^{*}}\left(\frac{\omega_{a}^{*}}{r^{*}} \frac{\partial \psi_{a}^{*}}{\partial z^{*}}\right)-\frac{\partial}{\partial z^{*}}\left(\frac{\omega_{a}^{*}}{r^{*}} \frac{\partial \psi_{a}^{*}}{\partial r^{*}}\right) \\
& +\operatorname{Pr}\left[\frac{\partial}{\partial r^{*}}\left(\frac{1}{r^{*}} \frac{\partial}{\partial r^{*}}\left(r^{*} \omega_{a}^{*}\right)\right)+\frac{\partial}{\partial z^{*}}\left(\frac{1}{r^{*}} \frac{\partial}{\partial z^{*}}\left(r^{*} \omega_{a}^{*}\right)\right)\right] \\
& \quad-\operatorname{Pr}\left(R a_{\mathrm{T}} \frac{\partial T_{a}^{*}}{\partial r^{*}}-R a_{\mathrm{v}} S_{\mathrm{v}}^{*}\right) \\
& \quad=0
\end{aligned}
$$

and

$S_{\mathrm{v}}^{*}=\frac{\partial w_{z}^{*}}{\partial z^{*}} \frac{\partial T_{a}^{*}}{\partial r^{*}}-\frac{\partial w_{z}^{*}}{\partial r^{*}} \frac{\partial T_{a}^{*}}{\partial z^{*}}$.

In the above equation, $w_{z}^{*}$ is the dimensionless axial pulsation velocity, which can be determined by the pulsation stream function $\psi_{\mathrm{w}}^{*}$ as follows:

$$
\frac{\partial}{\partial z^{*}}\left(\frac{1}{r^{*}} \frac{\partial \psi_{\mathrm{w}}^{*}}{\partial z^{*}}\right)+\frac{\partial}{\partial r^{*}}\left(\frac{1}{r^{*}} \frac{\partial \psi_{\mathrm{w}}^{*}}{\partial r^{*}}\right)-\frac{\partial T_{a}^{*}}{\partial r^{*}}=0 .
$$

Also, $\psi_{\mathrm{w}}^{*}$ is set to zero at boundaries. The pulsation velocities are related to the pulsation stream function $\psi_{\mathrm{w}}^{*}$ as:

$w_{r}^{*}=-\frac{1}{r^{*}} \frac{\partial \psi_{\mathrm{w}}^{*}}{\partial z^{*}}, \quad w_{z}^{*}=\frac{1}{r^{*}} \frac{\partial \psi_{\mathrm{w}}^{*}}{\partial r^{*}}$.

The dimensionless number $R a_{\mathrm{v}}$ in Eq. (10) is the vibrational Rayleigh number. It is defined as:

$$
R a_{\mathrm{v}} \equiv \frac{\left(\beta_{\mathrm{T}} b_{\mathrm{v}} \Omega_{\mathrm{v}} T_{\mathrm{m}} R_{\mathrm{c}}\right)^{2}}{2 \nu_{\mathrm{m}} \alpha_{\mathrm{m}}} .
$$

Since this dimensionless number is the only parameter appearing in Eq. (10) for thermovibration, it is clear that the averaged fields are independent of the vibration amplitude or frequency as long as $b_{\mathrm{v}} \Omega_{\mathrm{v}}$ is kept constant. In fact, such a result is only valid for the high-frequency limit. As will be shown shortly, the approximation breaks down for $f_{\mathrm{v}}<2 \mathrm{~Hz}$, where the resonance starts to kick in, which corresponds to a dimensionless angular frequency of about $3.6 \times 10^{3}$. Once the resonance exists, the high-frequency approximation gives an erroneous result. An interesting observation from both models is that the scaling relations for $\eta$ and $R a_{\mathrm{v}}$ are different; $b_{\mathrm{v}} \Omega_{\mathrm{v}}^{2}$ is for $\eta$ and $b_{\mathrm{v}}^{2} \Omega_{\mathrm{v}}^{2}$ for $R a_{\mathrm{v}}$. However, if the frequency is high enough, the result depends only on the scaling of $b_{\mathrm{v}} \Omega_{\mathrm{v}}$, not $b_{\mathrm{v}} \Omega_{\mathrm{v}}^{2}$.

Other time-averaged equations are in the same form as Eqs. (2) and (3). The suffix ' $a$ ' for the field variables is used to indicate the time-averaged properties. The solution scheme for the high-frequency model is also the same as the full approximation. However, since only the large time scale needs to be taken care, the

Table 1

Physical properties and some input parameters [24-26]

GaAs

$\rho_{\mathrm{c}}=\rho_{\mathrm{f}}=5.71 \mathrm{~g} \mathrm{~cm}^{-3}$ (assumed to be the same as $\rho_{\mathrm{m}}$ )

$\rho_{\mathrm{m}}=5.71 \mathrm{~g} \mathrm{~cm}^{-3}$

$\mu_{\mathrm{m}}=1.967 \times 10^{-2} \mathrm{~g} \mathrm{~cm}^{-1} \mathrm{~s}^{-1}$

$T_{\mathrm{m}}=1511 \mathrm{~K}$

$\Delta H=726 \mathrm{~J} \mathrm{~g}^{-1}$

$h=4.096 \times 10^{-4} \mathrm{~W} \mathrm{~cm}^{-2} \mathrm{~K}^{-1}$

$k_{\mathrm{c}}=k_{\mathrm{f}}=0.07 \mathrm{~W} \mathrm{~cm}^{-1} \mathrm{~K}^{-1}$

$k_{\mathrm{m}}=0.14 \mathrm{~W} \mathrm{~cm}^{-1} \mathrm{~K}^{-1}$

$C_{\mathrm{p}_{\mathrm{c}}}=C_{\mathrm{p}_{\mathrm{f}}}=C_{\mathrm{p}_{\mathrm{m}}}=0.42 \mathrm{~J} \mathrm{~g}^{-1} \mathrm{~K}^{-1}$

$\beta_{\mathrm{T}}=1.16 \times 10^{-4} \mathrm{~K}^{-1}$

Quartz (ampoule)

$\rho_{\text {amp }}=2.2 \mathrm{~g} \mathrm{~cm}^{-3}$

$k_{\text {amp }}=0.035 \mathrm{~W} \mathrm{~cm}^{-1} \mathrm{~K}^{-1}$

$C_{\text {pamp }}=0.188 \mathrm{~J} \mathrm{~g}^{-1} \mathrm{~K}^{-1}$

$\varepsilon_{\mathrm{amp}}=0.7$

Other input parameters

$L=15 \mathrm{~cm}$

$R_{\mathrm{c}}=1 \mathrm{~cm}$

$R_{\mathrm{amp}}=1.2 \mathrm{~cm}$

$T_{\mathrm{p}}=1800 \mathrm{~K}$

$T_{\mathrm{v}}=898 \mathrm{~K}$

$w=1.25 \mathrm{~cm}$

$a=0.2 \mathrm{~cm}$

$z_{\mathrm{p} 0}=7.5 \mathrm{~cm}$

$b_{\mathrm{v}}=0-10 \mathrm{~cm}$

$f_{\mathrm{v}}=0.1-100 \mathrm{~Hz}$

$U_{\text {amp }}=-1 \mathrm{~cm} / \mathrm{h}$ (for pseudo-steady state calculations)

$U_{\mathrm{h}}=0 \mathrm{~cm} / \mathrm{h}$ (for fully transint calculations)

Dimensionless groups

$\operatorname{Pr}=0.059 ; R a_{\mathrm{T}}=0-8.51 \times 10^{5} ; \eta=0-402.84 ;$

$R a_{\mathrm{v}}=0-3.01 \times 10^{8}$, 
stable integration stepsize can be much larger, and thus the computation effort is reduced dramatically.

It should be pointed out again that the difficulty for solving the full equations for the 'moving boundary' problem is much higher than that for the 'fixed boundary' one. For the moving boundary problem, the boundary shape is not relaxed until the temperature field reaches a steady state. In other words, the zone shape is determined by the large time scale (in the order of several minutes at least). Therefore, tremendous integration effort is required to reach the steady 'harmonic' state. Therefore, for getting a harmonic state, our strategy to reduce the computational cost is to use looser convergence criteria at the beginning. As the overall zone shape is approaching to a stable zone, which can be detected by the interface deflection, we are then tightening up the convergence criteria for integration.

\section{Results and discussion}

Before showing calculated results, we have performed extensive benchmark for the numerical simulation of VZM crystal growth [9,19] for both steady and unsteady states. Good agreement with the results obtained by using FLUENT, a commercial CFD package, has been obtained [9]. Detailed code verification can be found elsewhere as well [9,23], which will not repeated here. The physical properties and some input parameters used in the following calculations are listed in Table 1 [24,25], where some typical values for the dimensionless groups are given. Due to the temperature difference, the viscosity varies about $30 \%$ in the molten zone. Therefore, an averaged value is used [26]. Furthermore, to match the $R a_{\mathrm{T}}$ at normal gravity reported in Ref. [25], the thermal expansion value $\beta_{\mathrm{T}}$ is also adjusted for comparison purposes. In the following sections, we will first illustrate the effects of axial vibration at the high-frequency limit. The verification of the high-frequency model will then be discussed through the comparison with the full model. Furthermore, from the full model, the effects of frequency can then be examined, followed by the discussion of the effects on the interface rate oscillation.

\subsection{Effects of thermovibration: high-frequency limit}

When the vibration frequency is high enough, a harmonic growth state may be obtained. Through the nonlinear coupling of the oscillatory terms, the heat flow, in the time-average sense, due to thermovibration could be quite different from the typical buoyancy flow due to radial heating. To illustrate that, we pick up a steady zone-melting of GaAs for consideration, where the pseudo-steady state model can be used. The growth rate is set to be $1 \mathrm{~cm} / \mathrm{h}$ (or $U_{\text {amp }}=-1 \mathrm{~cm} / \mathrm{h}$ ). Fig. 2 shows the results at different thermal $\left(R a_{\mathrm{T}}\right)$ and vibrational $\left(R a_{\mathrm{v}}\right)$ Rayleigh numbers. First of all, let's examine the results due to the buoyancy convection $\left(R a_{\mathrm{v}}=0\right)$. They are shown from Fig. 2(a)-(c); $R a_{\mathrm{T}}=$ $8.51 \times 10^{5}$ corresponds to the normal gravity condition. As shown, under normal gravity the convective
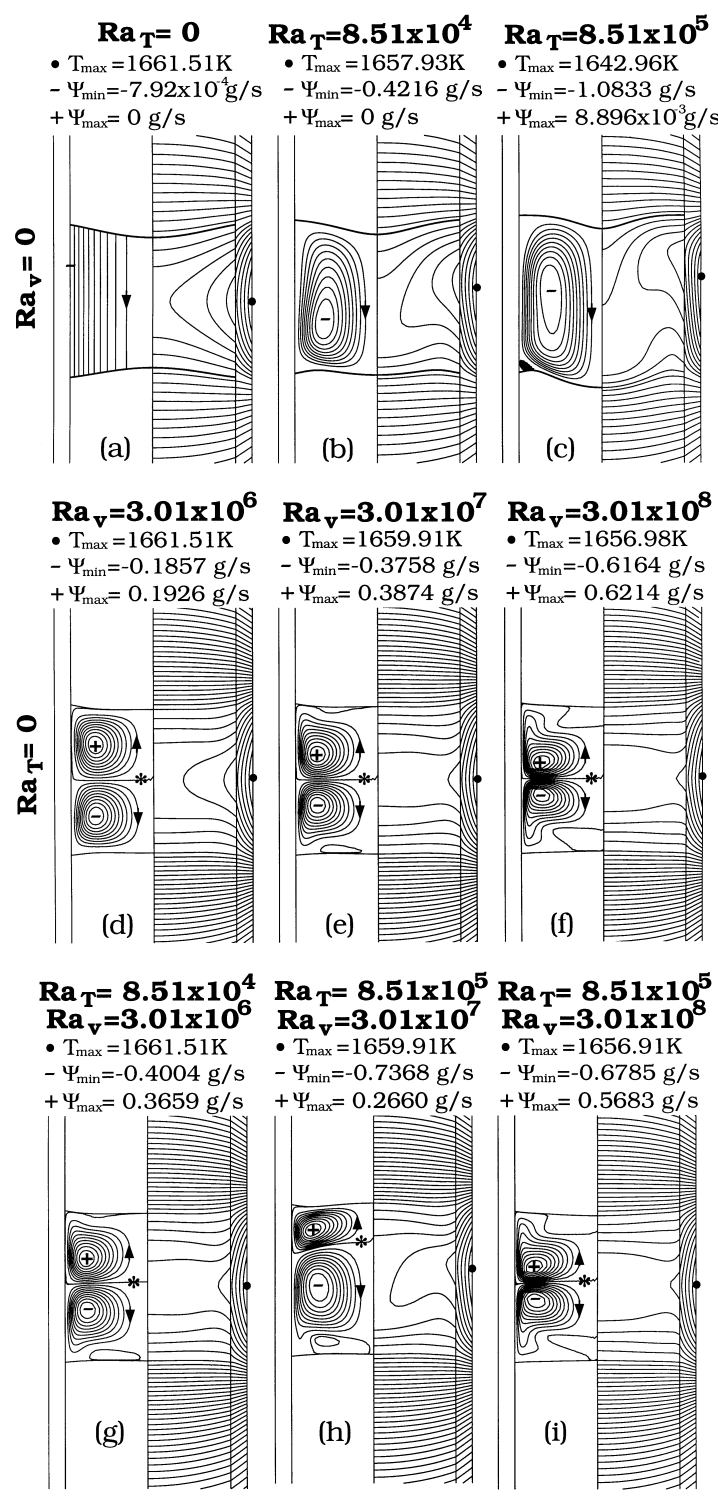

Fig. 2. Effects of buoyancy convection and vibration on the flow, thermal fields, and interface shapes. (a) $R a_{\mathrm{T}}=0$, $R a_{\mathrm{v}}=0$; (b) $R a_{\mathrm{T}}=8.51 \times 10^{4}, R a_{\mathrm{v}}=0$; (c) $R a_{\mathrm{T}}=8.51 \times 10^{5}$, $R a_{\mathrm{v}}=0$; (d) $R a_{\mathrm{v}}=3.01 \times 10^{6}, R a_{\mathrm{T}}=0$; (e) $R a_{\mathrm{v}}=3.01 \times 10^{7}$, $R a_{\mathrm{T}}=0$; (f) $R a_{\mathrm{v}}=3.01 \times 10^{8}, R a_{\mathrm{T}}=0 ;\left(\right.$ g) $R a_{\mathrm{v}}=3.01 \times 10^{6}$, $R a_{\mathrm{T}}=8.51 \times 10^{4}$; (h) $R a_{\mathrm{v}}=3.01 \times 10^{7}, R a_{\mathrm{T}}=8.51 \times 10^{5}$; (i) $R a_{\mathrm{v}}=3.01 \times 10^{8}, R a_{\mathrm{T}}=8.51 \times 10^{5} . U_{\mathrm{amp}}=-1 \mathrm{~cm} / \mathrm{h}$. 
heat transfer is significant in the melt zone. As a result, the growth interface can be inverted from convex to concave. As mentioned before, the concave interface shape (Fig. 2(c)) is not favorable in the growth of single crystals. Therefore, the growth situation in case Fig. 2(c) needs to be modified for the need of crystal growth. The maximum velocity in Fig. 2(c) is about $2.56 \mathrm{~cm} / \mathrm{s}$ at the centerline (near the middle of the zone), while it is about $2.7778 \times 10^{-4} \mathrm{~cm} / \mathrm{s}$ in Fig. 2(a) and $0.157 \mathrm{~cm} / \mathrm{s}$ in Fig. 2(b).

On the other hand, with the thermovibration alone, as shown from Fig. 2(d) and (e), two vortices are induced, and the flows are very different from that due to the buoyancy force shown in Fig. 2(c). As will be illustrated shortly, even for the time-dependent flow, the flow structures are similar in a period of vibration, but the flows are somewhat modified by the vibration. Clearly, with the axial vibration, near the heater, the melt is pumped inward from the zone surface to the zone center making the radial heating more efficient. As a result, the growth interface is flattened, but still convex. Such a growth interface is thus preferred for singe crystal growth. More importantly, since the thermovibrational convection is driven by the thermal gradients. As the isotherms are flattened horizontally, further increasing vibration does not affect much the flattened isotherms. This can be further understood by the source term of Eq. (10); $\partial T_{a} / \partial r$ is the driving force for the pulsation flow. Therefore, as shown, the interface shapes are not changed much even though $R a_{\mathrm{v}}$ has been increased by two orders of magnitude from Fig. 2(a)-(c). Nevertheless, further increasing $R a_{\mathrm{v}}$, on the contrary, induces secondary cells that have a different flow direction from the main cells. Although the secondary cell may not be significant enough to modify the local heat transfer due to the small Prandtl number $(P r=0.059)$, it may affect significantly the radial solute segregation due to the much larger Schmidt number for most impurities.

The maximum velocity for Fig. 2(d) is about 0.216 $\mathrm{cm} / \mathrm{s}$, while 0.528 and $1.188 \mathrm{~cm} / \mathrm{s}$ for Fig. 2(e) and (f), respectively. It should be pointed out that the vibration shown here, even for Fig. 2(d), is very severe. For the case of $1 \mathrm{kHz}$ vibration, the vibration amplitude ranges from 0.03162 to $0.3162 \mathrm{~cm}$ for cases Fig. 2(d)-(f); $b_{\mathrm{v}} f_{\mathrm{v}}=100 \mathrm{~cm} / \mathrm{s}$ for Fig. 2(e) and $316.2 \mathrm{~cm} / \mathrm{s}$ for Fig. 2(f). This is because the Prandtl number of GaAs is not very high that significant flow is necessary to affect the thermal field and thus the growth interface. In the horizontal Bridgman configuration studied by Uspenskii and Favier [15], $b_{\mathrm{v}} f_{\mathrm{v}}=10^{3} \mathrm{~cm} / \mathrm{s}$ is necessary to damp the convection, which is about one order of magnitude higher than our cases. Indeed, as we combine both the buoyancy and thermovibration modes, it is clearer that why such a strong vibration action is necessary.
The combined effects of two forces are illustrated from Fig. 2(g)-(i). Again, with vibration, the concave growth interface due to the buoyancy force is resolved, and the growth interface becomes very flat or slightly convex. As compared with cases Fig. 2(f) and (i), at $R a_{\mathrm{v}}=3.01 \times 10^{8}$, the effect of buoyancy force is barely seen. Accordingly, the buoyancy convection is suppressed, which is consistent with the observation by Uspenskii and Favier [15]. However, they applied the vibration horizontally with $b_{\mathrm{v}} f_{\mathrm{v}}=10^{3} \mathrm{~cm} / \mathrm{s}$ and the maximum melt velocity was reduced three times. In our case Fig. 2(i), $b_{\mathrm{v}} f_{\mathrm{v}}=316.2 \mathrm{~cm} / \mathrm{s}$, and the maximum velocity is reduced from $2.56 \mathrm{~cm} / \mathrm{s}$ in Fig. 2(c) to 1.188 $\mathrm{cm} / \mathrm{s}$ in Fig. 2(i). Interestingly, the maximum velocity for case Fig. 2(h) is about $1.2 \mathrm{~cm} / \mathrm{s}$. In other words, completely suppressing the buoyancy flow does not reduce much the maximum velocity because the vibration also generates its own flow. Furthermore, even though $R a_{\mathrm{v}} \approx 10^{8}$ may be the limit that could be reached in practice, our purpose here is not to totally suppress the buoyancy flow, but to obtain a convex interface for normal gravity growth. Therefore, the case Fig. 2(h) seems to be satisfactory for our application. However, the critical vibration for interface inversion is important and needs to be found.

The critical vibration can be found by tracing the solution using $R a_{\mathrm{v}}$ as a parameter. Fig. 3 shows the

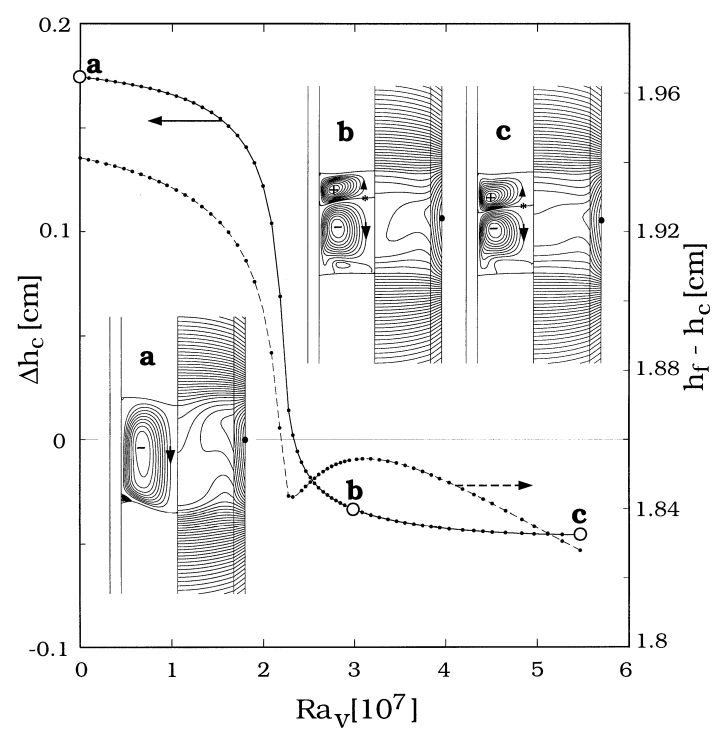

Fig. 3. Effect of axial vibration intensity $\left(R a_{\mathrm{v}}\right)$ on the interface deflection $\Delta h_{\mathrm{c}}\left(\Delta h_{\mathrm{c}}=\left.h_{\mathrm{c}}\right|_{R_{\mathrm{c}}}-\left.h_{\mathrm{c}}\right|_{r=0}\right)$, zone length $\left(h_{\mathrm{f}}-h_{\mathrm{c}}\right)$ at $r=0$, and heat flows. In the field plots, $\psi_{\min }=-1.078, \psi_{\max }=$ $9.547 \times 10^{-3} \mathrm{~g} / \mathrm{s}$, and $T_{\max }=1643.09 \quad \mathrm{~K}$ for $\mathrm{a}$; $\psi_{\min }=-0.7403, \psi_{\max }=0.2636 \mathrm{~g} / \mathrm{s}$, and $T_{\max }=1657.01 \mathrm{~K}$ for $\mathrm{b} ; \psi_{\min }=-0.6648, \psi_{\max }=0.3198 \mathrm{~g} / \mathrm{s}$, and $T_{\max }=1657.81 \mathrm{~K}$ for $\mathrm{c}$. 
effects of vibration on the interface deflection $\left(\Delta h_{\mathrm{c}}=\right.$ $\left.\left.h_{\mathrm{c}}\right|_{r=R_{\mathrm{c}}}-\left.h_{\mathrm{c}}\right|_{r=0}\right)$ and the zone length. From there, the critical $R a_{\mathrm{v}}$ (about $2.2 \times 10^{7}$ ) can be found, where the interface inversion takes place. For GaAs at this value, $b_{\mathrm{v}} f_{\mathrm{v}}$ is about $70 \mathrm{~cm} / \mathrm{s}$; for $f_{\mathrm{v}}=1 \mathrm{kHz}$, the vibration amplitude is about $0.7 \mathrm{~mm}$. After the dramatic change, the interface becomes flat. As just explained, the interface deflection is not changed much by further increasing $R a_{\mathrm{v}}$ due to the small radial thermal gradient near the growth interface. Nevertheless, again, the newly formed secondary cell near the growth interface, as shown in cases Fig. 2(b) and (c), can play an important role in the local mass transfer leading to different radial segregations.

\subsection{Validity of high-frequency approximation}

In the previous section, the effect of axial vibration is illustrated for the high-frequency limit. Now, the next question is the validity of such an approximation. Furthermore, since $b_{\mathrm{v}} f_{\mathrm{v}}$ or $R a_{\mathrm{v}}$ is the only parameter in the high-frequency model, the frequency does not affect the result if $R a_{\mathrm{v}}$ is kept the same. However, in the full model, the parameters that really matters are the relative amplitude of gravity modulation $\eta$, or $b_{\mathrm{v}} f_{\mathrm{v}}^{2}$, and $f_{\mathrm{v}}$. Therefore, keeping the same $b_{\mathrm{v}} f_{\mathrm{v}}$ in the high-frequency limit should have a time-average result that is independent of frequency. Therefore, to validate the high-frequency model used in this paper, we have performed a series calculations using the full model. After a harmonic result is obtained for all variables, we then take a time average of the variables for comparison.

Two cases are considered using $10 \mathrm{~Hz}$ axial vibration for a stationary zone $\left(U_{\mathrm{amp}}=U_{\mathrm{h}}=0\right)$; one is with the thermovibration alone $\left(R a_{\mathrm{v}}=3.01 \times 10^{7}\right.$ or $\left.\eta=40.284, R a_{\mathrm{T}}=0\right)$ and the other also incorporates the effects of buoyancy force $\left(R a_{\mathrm{v}}=3.01 \times 10^{7}\right.$, $\left.R a_{\mathrm{T}}=8.51 \times 10^{5}\right)$. For $10 \mathrm{~Hz}$ vibration, the amplitude used is $10 \mathrm{~cm}$ here. In practice, it may be difficult to vibrate the system with such a high amplitude. However, the high $R a_{\mathrm{v}}$ used in Fig. 2(h) is proven just enough for interface inversion. Therefore, to keep the same $R a_{\mathrm{v}}$, if we use a smaller amplitude, the frequency needs to be increased, which increases the computational effort significantly for the full model to get a harmonic result. The system will take at least 20 to 30 min for the initial transient period to be relaxed. In addition, as will be discussed later, $10 \mathrm{~Hz}$ is high enough for the high-frequency limit to be achieved. Therefore, using a higher frequency to examine the high-frequency limit is prohibited here. As a tradeoff, we thus pick $10 \mathrm{~Hz}$ to validate our high-frequency model here; $100 \mathrm{~Hz}$ has also been used for further testing, and the conclusion is the same.
After the initial transient period is removed, Fig. 4 shows the steady oscillation pattern of the thermovibration flow at $R a_{\mathrm{v}}=3.01 \times 10^{7} ; R a_{\mathrm{T}}=0$. The thermal and flow fields at the last period are also illustrated. As shown, even the frequency is high, the flow structures still can respond periodically with time. Within one vibration period, the size change of each cell is clear. Even so, the two-cell flow structure still remains and the change of the interface shape is not obvious. If we take the last 5 periods for computing the averaged flow field, we can make a comparison with the results from the high-frequency model (with $U_{\text {amp }}=0$ ). As shown in Fig. 5, the agreement on the flow patterns appears to be excellent; the streamlines are obtained with the interval of $\left(\psi_{\max }-\psi_{\min }\right) / 20$ for both plots. Besides the nearly-zero streamline, the other streamlines are almost overlapped to each other. Nevertheless, one should not forget that the actual flow fields do change periodically with the vibration, as illustrated in Fig. 4. Since the gravity effect is removed, the upper and lower flow cells shown in Fig. 5 are symmetric to each other. From the application point of view, if we don't care much about the oscillation of heat flow, the time-average result obtained from the high-frequency model seems to be satisfactory. The effect on the interface shapes can also be well predicted.

The case with the buoyancy force $\left(R a_{\mathrm{T}}=8.51 \times 10^{5}\right)$ also agrees well with the high-frequency limit. The har-

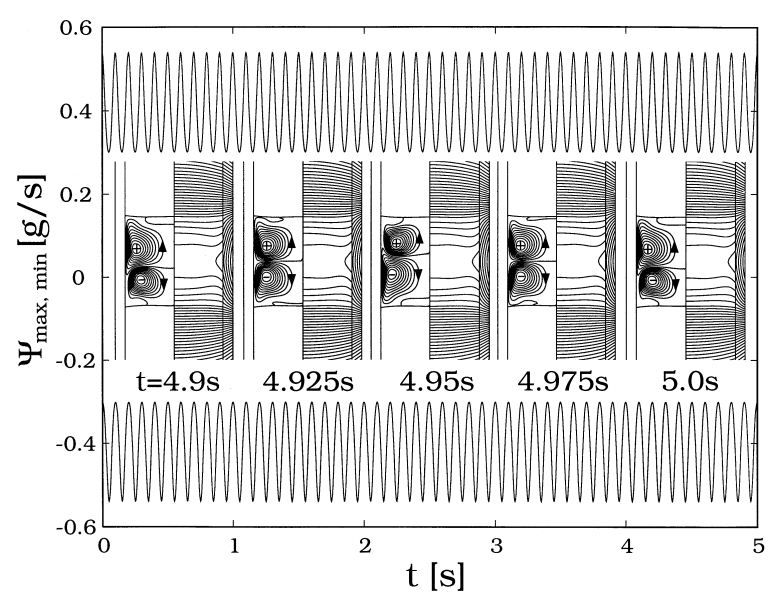

Fig. 4. Harmonic solution due to axial vibration for $R a_{\mathrm{T}}=0$ and $R a_{\mathrm{v}}=3.01 \times 10^{7}$. Some flow patterns and isotherms at the last period are also illustrated. $\psi_{\min }=-0.3012$, $\psi_{\max }=0.5387 \mathrm{~g} / \mathrm{s}$, and $T_{\max }=1659.43 \mathrm{~K}$ for $t=4.9 \mathrm{~s}$; $\psi_{\min }=-0.3968, \psi_{\max }=0.3847 \mathrm{~g} / \mathrm{s}$, and $T_{\max }=1659.43 \mathrm{~K}$ for $t=4.925 \quad \mathrm{~s} ; \quad \psi_{\min }=-0.5388, \quad \psi_{\max }=0.3012 \mathrm{~g} / \mathrm{s}, \quad$ and $T_{\max }=1659.43 \quad \mathrm{~K} \quad$ for $t=4.95 \quad \mathrm{~s} ; \quad \psi_{\min }=-0.3847$, $\psi_{\max }=0.3967 \mathrm{~g} / \mathrm{s}$, and $T_{\max }=1659.43 \mathrm{~K}$ for $t=4.975 \mathrm{~s}$; $\psi_{\min }=-0.3012, \psi_{\max }=0.5387 \mathrm{~g} / \mathrm{s}$, and $T_{\max }=1659.43 \mathrm{~K}$ for $t=5 \mathrm{~s}$. 
$\mathrm{Ra}_{\mathrm{v}}=3.01 \times 10^{7} ; \mathrm{Ra}_{\mathrm{T}}=0$

\section{High-frequency Time-avereged approximation flow field}

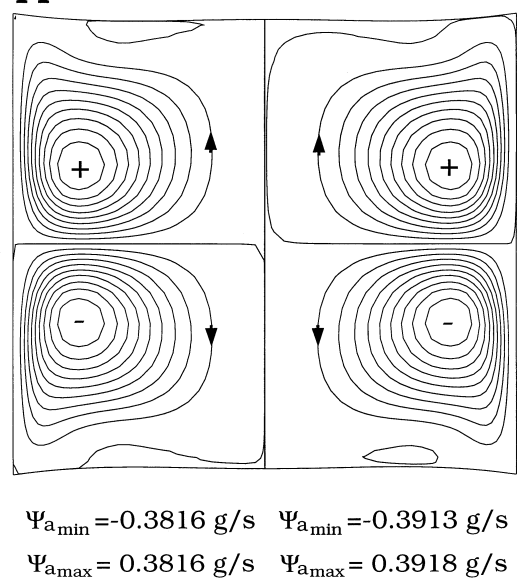

Fig. 5. Comparison of averaged flow patterns from Fig. 4 with that obtained from the high-frequency model.

monic oscillation of the flow intensity is shown in Fig. 6. As shown by the flow fields at the last period, because of the buoyancy convection, the flow structure becomes asymmetric; the upper cell is smaller. More interestingly, a tiny cell just above the growth interface

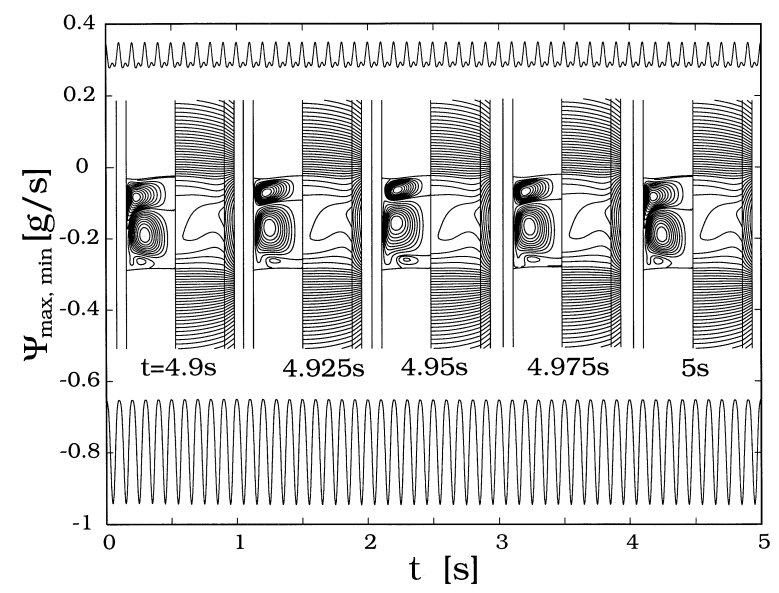

Fig. 6. Harmonic solution due to axial vibration for $R a_{\mathrm{T}}=8.51 \times 10^{5}$ and $R a_{\mathrm{v}}=3.01 \times 10^{7}$. Some flow patterns and isotherms at the last period are also illustrated. $\psi_{\min }=-0.6536, \psi_{\max }=0.3475 \mathrm{~g} / \mathrm{s}$, and $T_{\max }=1657.23 \mathrm{~K}$ for $t=4.9 \quad \mathrm{~s} ; \quad \psi_{\min }=-0.7492, \quad \psi_{\max }=0.2803 \quad \mathrm{~g} / \mathrm{s}, \quad$ and $T_{\max }=1657.23 \quad \mathrm{~K} \quad$ for $t=4.925 \quad \mathrm{~s} ; \quad \psi_{\min }=-0.9444$, $\psi_{\max }=0.2927 \mathrm{~g} / \mathrm{s}$, and $T_{\max }=1657.23 \mathrm{~K}$ for $t=4.95 \mathrm{~s}$; $\psi_{\min }=-0.7352, \psi_{\max }=0.2873 \mathrm{~g} / \mathrm{s}$, and $T_{\max }=1657.24 \mathrm{~K}$ for $t=4.975 \quad \mathrm{~s} ; \quad \psi_{\min }=-0.6536, \quad \psi_{\max }=0.3474 \mathrm{~g} / \mathrm{s}, \quad$ and $T_{\max }=1657.23 \mathrm{~K}$ for $t=5 \mathrm{~s}$. moves around periodically with vibration. As just mentioned, such an unstable cell may also be responsible on the local mass transfer leading to growth striations. The time average of the flow patterns in the last five periods is shown in Fig. 7. Again, it is in good agreement with the high-frequency approximation. Therefore, it may be safe to conclude that for high vibration frequency (at least $10 \mathrm{~Hz}$ for now), the high-frequency model is satisfactory in the averaged flow and the interface shapes. Furthermore, from the comparison with the full model, the Schlichting effect, which is ignored in our high-frequency model, is small. Again, as mentioned before, the vibration amplitude of the interface is much smaller than the applied one. Nevertheless, the onset of resonance and interface oscillation still remain a great interest to crystal growers. Therefore, they need to be discussed in details.

\subsection{Effects of vibration frequency: resonance}

As the vibration is utilized in practice, the vibration frequency is a key parameter that needs to be decided carefully. We have examined the frequency ranging from 0.1 to $100 \mathrm{~Hz}$ using the full model. Through the comparison with the high-frequency model using the same $R a_{\mathrm{v}}$, we can examine the breakdown of the highfrequency model and the onset of resonance. The effect of frequency on the average flow intensities is summarized in Fig. 8. The amplitude of flow oscillation is also indicated by the error bars. As shown, if the frequency is high enough, the high-frequency limit can be

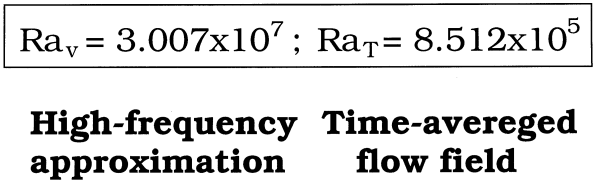

\section{High-frequency Time-avereged approximation flow field}

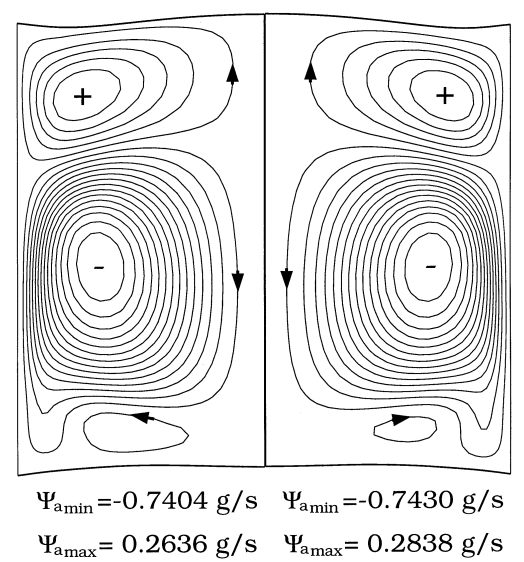

Fig. 7. Comparison of averaged flow patterns from Fig. 6 with that obtained from the high-frequency model. 
approached. For $f_{\mathrm{v}}>5 \mathrm{~Hz}$, the high-frequency model can be a good approximation. Furthermore, because $R a_{\mathrm{v}}$ (or $b_{\mathrm{v}} f_{\mathrm{v}}$ ) is kept the same, the oscillation amplitude of the flow approaches to a constant at high frequency. On the other hand, at lower frequency $(<2$ $\mathrm{Hz}$ ) the high-frequency model starts to break down. One can easily find the breakdown from the averaged flow patterns illustrated on the top of the figure. As shown, for $f_{\mathrm{v}}<2 \mathrm{~Hz}$, the flow fields becomes very different. The calculated zone shapes are very different as well. Interestingly, the flow intensity of the main cell increases dramatically near $0.5 \mathrm{~Hz}$ indicating the onset of resonance; the oscillation amplitude increases as well. Further decreasing the frequency, the effect of resonance decreases.

More importantly, the resonance has a profound effect on the oscillation amplitude of the interface speed, as shown in Fig. 9. Near $0.5 \mathrm{~Hz}$, there is a maximum on the oscillation amplitude. A typical crystal growth rate, $1 \mathrm{~cm} / \mathrm{h}$, is also indicated in the same figure. As shown, for $f_{\mathrm{v}}<5 \mathrm{~Hz}$, severe periodic growth and remelting could appear. Therefore, higher frequency $(5 \mathrm{~Hz})$ is necessary to reduce the growth fluctuation.

Indeed, as shown by the results so far, although the high-frequency model works quite well for the frequency ranging from 2 to $100 \mathrm{~Hz}$ (and higher of course), it does not provide any information on the oscillation amplitude of the variables in the system. The fluctuation amplitude of the interface speed, as well as

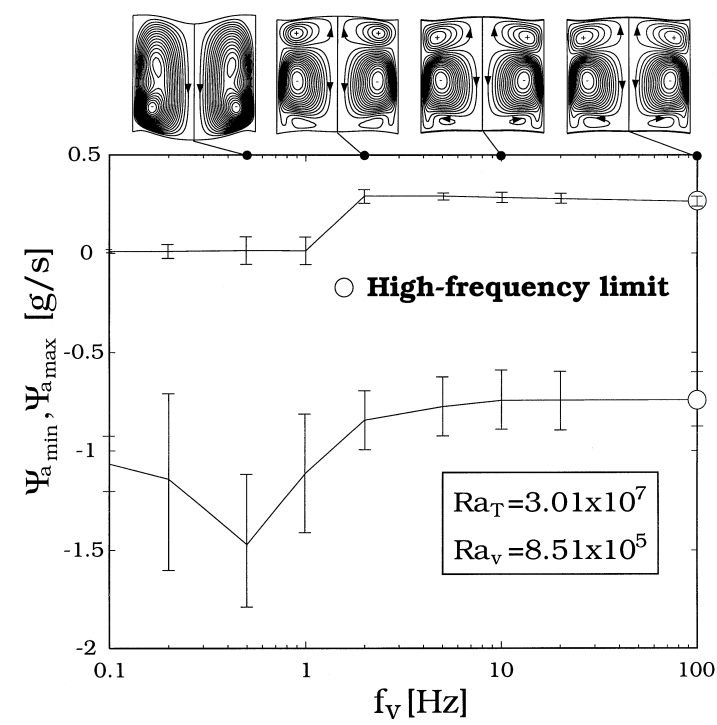

Fig. 8. Effect of vibration frequency on the flows and flow intensity; $R a_{\mathrm{T}}=8.51 \times 10^{5}$ and $R a_{\mathrm{v}}=3.01 \times 10^{7}$. The error bars indicate the amplitude of oscillation. The averaged flow patterns at $0.5,2,10$, and $100 \mathrm{~Hz}$ are shown on the top of the figure.

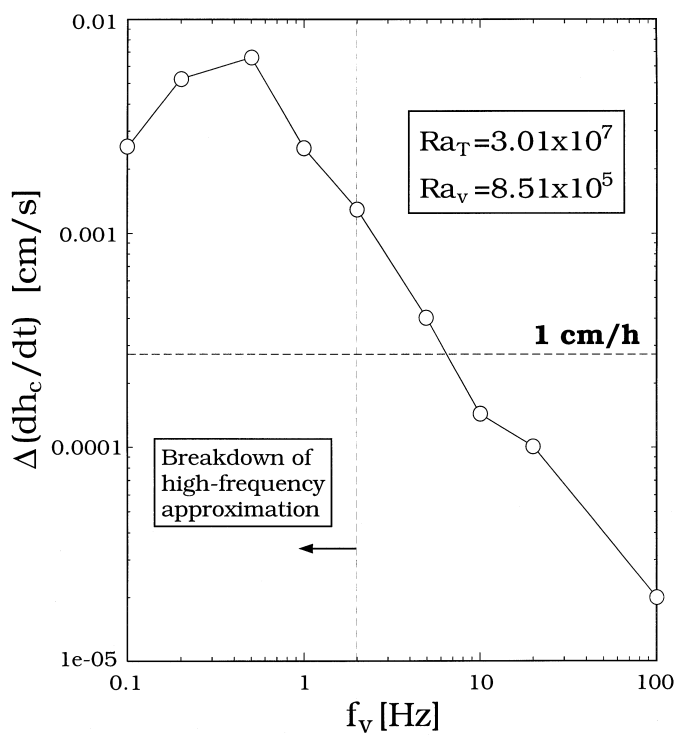

Fig. 9. Effect of vibration frequency on the amplitude of oscillation of the interface speed; $R a_{\mathrm{T}}=8.51 \times 10^{5}$ and $R a_{\mathrm{v}}=3.01 \times 10^{7}$. The dashed line at $1 \mathrm{~cm} / \mathrm{h}$ indicates a typical interface speed for crystal growth.

the flow intensity, is a key issue that needs to be considered in practice. Fig. 9 clearly indicates the significance of the vibration frequency on the growth rate fluctuation. For crystal growth applications, it is clear that the higher the frequency the better it is. However, as implemented in practice, there are other problems, such as the mechanical design and flow instability, that need to be considered as well.

\section{Conclusions}

The effects of axial vibration on the heat flow, the growth rate, and the interface shapes are studied numerically for vertical zone-melting. The results provide some insight on the use of vibration on the process control, especially, on the zone shape and the flows. It is found that the axial vibration can be effective for the control of melt flows and the growth interface, but the critical vibration is not trivial. At high frequency, the minimum vibration speed $\left(b_{\mathrm{v}} f_{\mathrm{v}}\right)$ is about $70 \mathrm{~cm} / \mathrm{s}$ or $R a_{\mathrm{v}} \approx 2.2 \times 10^{7}$. For $2-\mathrm{cm} \mathrm{GaAs,} \mathrm{the} \mathrm{high-frequency}$ model may be used for the frequency greater than 2 Hz. At lower frequency, the model starts to break down, and the resonance appears at about $0.5 \mathrm{~Hz}$. Away from the frequency, the fluctuation of the interface speed decreases.

To prevent crystal growth from periodic remelting, a frequency higher than $10 \mathrm{~Hz}$ seems to be necessary. Nevertheless, as the frequency is increased further, the 
flow oscillation decays very slowly. So far, the real applications of vibration on crystal growth or purification seem to be rare. However, from our calculations, we believe that the axial vibration may be a useful way to control zone-melting, but this still requires further experimental verification in the future.

\section{References}

[1] J.C. Brice, Crystal Growth Processes, Wiley, New York, 1986, p. 112.

[2] C.W. Lan, C.C. Ting, Computer simulation of liquid encapsulated vertical Bridgman crystal growth, Int. J. Numerical Methods in Heat Transfer and Fluid Flow 6 (1996) 3-24.

[3] D. Hofmann, T. Jung, G. Muller, Growth of 2 inch Ge:Ga crystals by the dynamic vertical gradient freeze process and its numerical modeling including transient segregation, J. Crystal Growth 128 (1993) 213-218.

[4] H.P. Utech, M.C. Flemming, Elimination of solute banding in indium atimonide crystals by growth in a magnetic field, J. Appl. Phys 37 (1966) 2021-2034.

[5] K.M. Kim, Suppression of thermal convection by transverse magnetic field, J. Electrochem. Soc 132 (1982) 427-437.

[6] D.H. Kim, P.M. Adornato, R.A. Brown, Effect of vertical magnetic field on convection and segregation in vertical Bridgman crystal growth, J. Crystal Growth 89 (1988) 339-356.

[7] C.W. Lan, Effects of ampoule rotation on the flows and dopant segregation in vertical Bridgman crystal growth, J. Crystal Growth 197 (1999) 983-991.

[8] H.J. Scheel, Accelerated crucible rotation: a novel stirring technique in high-temperature solution growth, J. Crystal Growth 13/14 (1971) 560-565.

[9] C.W. Lan, J.H. Chian, Effects of ampoule rotation on vertical zone-melting crystal growth: steady rotation versus accelerated crucible rotation technique (ACRT), J. Crystal Growth 203 (1999) 286-296.

[10] A.V. Anilkumar, R.N. Grugel, R.N. Shen, T.G. Wang, Control of thermocapillary convection in a liquid bridge by vibration, J. Applied Physics 73 (1993) 4165-4170.
[11] D.V. Lyubimov, T.P. Lyubimova, S. Meradji, B. Roux, Vibrational control of crystal growth from liquid phase, J. Crystal Growth 180 (1997) 648-659.

[13] W.S. Liu, M.F. Wolf, D. Elwell, R.S. Feigelson, Low frequency vibrational stirring: a new method for radial mixing solutions and melts during growth, J. Crystal Growth 82 (1987) 589-597.

[14] W. Yuan, M. Banan, L.L. Regel, W.R. Wilcox, The effect of vertical vibration of the ampoule on the direction solidification of InSb-GaSb alloy, J. Crystal Growth 151 (1995) 235-242.

[15] V. Uspenskii, J.J. Favier, High frequency vibration and natural convection in Bridgman-scheme crystal growth, Int. J. Heat and Mass Transfer 37 (1994) 691-698.

[16] G.K. Batchelor, An Introduction to Fluid Dynamics, Cambridge University Press, Cambridge, UK, 1967, pp. 358-360.

[17] D.V. Lyubimov, T.P. Lyubimova, B. Roux, Mechanisms of vibrational control of heat transfer in a liquid bridge, Int. J. Heat Mass Transfer 40 (1997) 4031-4042.

[18] W.G. Pfann, Zone Melting, 2nd ed., Wiley, New York, 1966.

[19] C.W. Lan, D.T. Yang, Dynamic simulation of the vertical zone-melting crystal growth, Int. J. Heat Mass Transfer 41 (1998) 4351-4373.

[20] F.V. Wald, R. O Bell, Natural and forced convection during solution growth by the travelling heater method (THM), J. Crystal Growth 30 (1975) 29-36.

[21] P.N. Brown, A.C. Hindmarsh, L.R. Petzold, Using Krylov methods in the solution of large-scale differential-algebraic systems, SIAM J. Sci. Comput 15 (1994) 1467-1487.

[22] G.Z. Gershuni, D.V. Lyubimov, Thermal Vibration Convection, Wiley, New York, 1998, pp. 2-7.

[23] C.W. Lan, D.T. Yang, C.C. Ting, F.C. Chen, A transparent furnace for crystal growth and flow visualization, J. Crystal Growth 142 (1994) 373-378.

[24] J.J. Derby, R.A. Brown, J. Crystal Growth 74 (1986) 605-624

[25] D.H. Kim, R.A. Brown, J. Crystal Growth 109 (1991) 66-74.

[26] K. Kakimoto, T. Hibiya, Appl. Phys. Lett 50 (18) (1987) 1249-1250. 